\title{
Two-Weight Extrapolation on Lorentz Spaces
}

\author{
Wenming Li, ${ }^{1}$ Tingting Zhang, ${ }^{1}$ and Limei Xue ${ }^{2}$ \\ ${ }^{1}$ College of Mathematics and Information Science, Hebei Normal University, Shijiazhuang, Hebei 050024, China \\ ${ }^{2}$ School of Mathematics and Science, Shijiazhuang University of Economics, Shijiazhuang 050031, China
}

Correspondence should be addressed to Wenming Li; lwmingg@sina.com

Received 24 October 2014; Accepted 17 December 2014

Academic Editor: Stanislav Hencl

Copyright (C) 2015 Wenming Li et al. This is an open access article distributed under the Creative Commons Attribution License, which permits unrestricted use, distribution, and reproduction in any medium, provided the original work is properly cited.

We give several two-weight extrapolation theorems on Lorentz spaces which extend the results of Cruz-Uribe and Pérez (2000) and some applications for the singular integral operators, the potential type operators, and commutators.

\section{Introduction and Main Results}

The classical extrapolation theorem is due to Rubio de Francia [1], who showed that if sublinear operator $T$ is bounded on $L^{p_{0}}(w)$ for some $p_{0}, 1 \leq p_{0}<\infty$, and every $w \in A_{p_{0}}$, then $T$ is bounded on $L^{p}(w)$ for every $p, 1<p<\infty$ and every $w \in$ $A_{p}$. Cruz-Uribe et al. [2] and Curbera et al. [3] generalized the extrapolation theorem of Rubio de Francia and got a lot of new extrapolation theorems associated with $A_{\infty}$ weights. These theorems have proved to be the key to solving many problems in harmonic analysis.

Cruz-Uribe and Pérez [4] gave several other extrapolation theorems for pairs of weights of the forms $\left(w, M^{k} w\right)$ and $\left(w,(M w / w)^{r} w\right)$, where $w$ is any nonnegative function, $r>$ 1 , and $M^{k}$ is the $k$ th iteration of the Hardy-Littlewood maximal operator. The purpose of this paper is to extend the extrapolation theorems in [4] and derive some extrapolation results on Lorentz spaces.

Let $w(x), x \in \mathbb{R}^{n}$, be a nonnegative, locally integrable weight function. For a measurable function $f$,

$$
\lambda_{f}^{w}(t)=w\left\{x \in \mathbb{R}^{n}:|f(x)|>t\right\}, \quad t \geq 0
$$

is the distribution function of $f$ with respect to the measure $w(x) d x$. For $0<p, q \leq \infty$, the weighted Lorentz spaces
$L^{p, q}(w)$ are the collection of all functions $f$, such that $\|f\|_{L^{p, q}(w)}<\infty$, where

$$
\|f\|_{L^{p, q}(w)}= \begin{cases}\left(q \int_{0}^{\infty} \lambda_{f}^{w}(t)^{q / p} t^{q-1} d t\right)^{1 / q}, & 0<q<\infty, \\ \sup _{t>0} t \lambda_{f}^{w}(t)^{1 / p}, & q=\infty .\end{cases}
$$

For $1 \leq p<\infty, p^{\prime}$ denote the conjugate exponent of $p$ : $1 / p+1 / p^{\prime}=1$.

In the case when either $1<p<\infty$ and $1 \leq q \leq \infty$, or $p=q=1$, or $p=q=\infty, L^{p, q}(w)$ is a Banach space. Furthermore, we have the relationship

$$
\begin{aligned}
C_{1}\|f\|_{L^{p, q}(w)} & \leq \sup _{\|g\|_{L^{p^{\prime}, q^{\prime}}(w)} \leq 1}\left|\int_{\mathbb{R}^{n}} f(x) g(x) w(x) d x\right| \\
& \leq C_{2}\|f\|_{L^{p, q}(w)} .
\end{aligned}
$$

Our main results are the following.

Theorem 1. Let $S$ and $T$ be operators (not necessarily linear), and let $f$ be a function in a suitable test class for both $S$ and T. Suppose that there exist positive constants $p_{0}$ and $C_{0}$ and $a$ positive integer $k$ such that for all weights $w$

$$
\|T f\|_{L^{p_{0}(w)}} \leq C_{0}\|S f\|_{L^{p_{0}}\left(M^{k} w\right)} .
$$


Then, for all $p, p_{0}<p<\infty, p_{0} \leq q \leq \infty$, there exists a constant $C_{p}$ depending only on $C_{0}, p, p_{0}, k$, and $n$, such that, for all weights $w$,

$$
\|T f\|_{L^{p, q}(w)} \leq C_{p}\|S f\|_{L^{p, q}\left(M^{\left[k p / p_{0}\right]+1} w\right)},
$$

where $\left[k p / p_{0}\right]$ is the largest integer less than or equal to $k p / p_{0}$.

Theorem 2. Let $S$ and $T$ be operators (not necessarily linear), and let $f$ be a function in a suitable test class for both $S$ and $T$. Suppose that there exist positive constants $p_{0}$ and $C_{0}$ and a positive integer $k$ such that for all weights $w$

$$
\|T f\|_{L^{p_{0}(w)}} \leq C_{0}\|S f\|_{L^{p_{0}}(M w)} .
$$

Then, for all $p, p_{0}<p<\infty, p_{0} \leq q \leq \infty$, there exists a constant $C_{p}$ depending only on $C_{0}, p, p_{0}, k$, and $n$, such that, for all weights $w$,

$$
\|T f\|_{L^{p, q}(w)} \leq C_{p}\|S f\|_{L^{p, q}\left((M w / w)^{\left[p / p_{0}\right]} M w\right)} .
$$

\section{Applications}

Let $M$ be the Hardy-Littlewood maximal operator; Fefferman and Stein [5] showed that, for every $p, 1<p<\infty$, every nonnegative function $w$, and every function $f$,

$$
\int_{\mathbb{R}^{n}}|M f(x)|^{p} w(x) d x \leq C \int_{\mathbb{R}^{n}}|f(x)|^{p} M w(x) d x .
$$

Using Theorem 1, we can get the following result.

Proposition 3. For $p, 1<p<\infty, 1 \leq q \leq \infty$, there exists a constant $C>0$, such that, for all weights $w$ and function $f$,

$$
\|M f\|_{L^{p, q}(w)} \leq C\|f\|_{L^{p, q}(M w)} .
$$

Let $T$ be a Calderón-Zygmund operator. Wilson [6] and Pérez [7] showed that if $1<p<\infty$, then, for any weight $w$ and every $f$,

$$
\int_{\mathbb{R}^{n}}|T f(x)|^{p} w(x) d x \leq C \int_{\mathbb{R}^{n}}|f(x)|^{p} M^{[p]+1} w(x) d x,
$$

where the exponent $[p]+1$ is sharp. Using Theorem 1 we can get the following result.

Proposition 4. Let $T$ be a Calderón-Zygmund operator, $1<$ $p<\infty, 1 \leq q \leq \infty$; then, for any weight $w$ and every $f$,

$$
\|T f\|_{L^{p, q}(w)} \leq C\|f\|_{L^{p, q}\left(M^{[p]+1} w\right)} .
$$

For the commutators of singular integral operators with a BMO function $[T, b]$, Pérez $[8]$ showed the analogous weighted inequalities as in (10). By Theorem 1, we can give similar results as in Proposition 4; details are omitted.

Lerner [9] establishes

$$
\int_{\mathbb{R}^{n}}|T f(x)| w(x) d x \leq C \int_{\mathbb{R}^{n}} M f(x) M w(x) d x,
$$

for any Calderón-Zygmund operator $T$ and any arbitrary weight $w$. Using Theorems 1 and 2, we can get the following results.
Proposition 5. Let $T$ be a Calderón-Zygmund operator, $1<$ $p<\infty, 1 \leq q \leq \infty$; then, for any weight $w$ and all $f$,

$$
\begin{gathered}
\|T f\|_{L^{p, q}(w)} \leq C\|M f\|_{L^{p, q}\left(M^{[p]} w\right)}, \\
\|T f\|_{L^{p, q}(w)} \leq C\|M f\|_{L^{p, q}\left((M w / w)^{[p]} M w\right)} .
\end{gathered}
$$

Now, we consider the potential type operators and commutators. For a nonnegative, locally integrable function $\Phi$ on $\mathbb{R}^{n}$, assume that $\Phi$ satisfies the following weak growth condition: there are constants $\delta, c>0,0 \leq \varepsilon<1$ with the property that, for all $k \in \mathbb{Z}$,

$$
\sup _{2^{k}<|x| \leq 2^{k+1}} \Phi(x) \leq \frac{c}{2^{k n}} \int_{\delta(1-\varepsilon) 2^{k}<|y| \leq 2 \delta(1+\varepsilon) 2^{k}} \Phi(y) d y .
$$

The basic examples are provided by the Riesz potential of order $\alpha, I_{\alpha}$, defined by the kernel $\Phi(x)=|x|^{\alpha-n}, 0<\alpha<n$.

Define the potential type operator $T_{\Phi}$ and the maximal operator $M_{\widetilde{\Phi}}$ by

$$
\begin{gathered}
T_{\Phi} f(x)=\int_{\mathbb{R}^{n}} \Phi(x-y) f(y) d y, \\
M_{\widetilde{\Phi}} f(x)=\sup _{x \in Q} \frac{\widetilde{\Phi}(l(Q))}{|Q|} \int_{Q}|f(x)| d x,
\end{gathered}
$$

where

$$
\widetilde{\Phi}(t)=\int_{|z|<t} \Phi(z) d z, \quad t \geq 0 .
$$

For the Riesz potential of order $\alpha, \widetilde{\Phi}(t)=t^{\alpha}$. Pérez [10] studies the two-weight strong type $(p, q)$ inequalities for $T_{\Phi}, 1<p \leq$ $q<\infty$.

Lemma 6 (see [10]). Let $T_{\Phi}$ be the potential type operator with $\Phi$ satisfying (14), let $f$ and $g$ be bounded functions with compact support, and let $a>2^{n}$; then, there exist a family of cubes $Q_{k, j}$ and a family of pairwise disjoint subsets $E_{k, j}$, $E_{k, j} \subset Q_{k, j}$, with $\left|Q_{k, j}\right|<\left(1-2^{n} / a\right)^{-1}\left|E_{k, j}\right|$ for all $k, j$, such that

$$
\begin{aligned}
& \int_{\mathbb{R}^{n}} \mid T_{\Phi} f(x) g(x) \mid d x \\
& \leq C \sum_{k, j} \frac{1}{\left|3 Q_{k, j}\right|} \int_{3 Q_{k, j}} f(x) d x \frac{\widetilde{\Phi}\left(l\left(\rho Q_{k, j}\right)\right)}{\left|\rho Q_{k, j}\right|} \\
& \quad \times \int_{\rho Q_{k, j}} g(x) d x\left|E_{k, j}\right|,
\end{aligned}
$$

where $\rho=\delta(1+\varepsilon)$.

By Lemma 6, we can easily prove the following result.

Lemma 7. Let $T_{\Phi}$ be the potential type operator with $\Phi$ satisfying (14); then, there is a constant $C>0$ such that, for any weight $w$ and all $f$,

$$
\int_{\mathbb{R}^{n}}\left|T_{\Phi} f(x)\right| w(x) d x \leq C \int_{\mathbb{R}^{n}} M_{\widetilde{\Phi}} f(x) M w(x) d x .
$$


Using (18) and Theorems 1 and 2, we can get the following results.

Proposition 8. Let $T_{\Phi}$ be the potential type operator with $\Phi$ satisfying (14), $1<p<\infty, 1 \leq q \leq \infty$; then, for any weight $w$ and every $f$,

$$
\begin{gathered}
\left\|T_{\Phi} f\right\|_{L^{p, q}(w)} \leq C\left\|M_{\widetilde{\Phi}} f\right\|_{L^{p, q}\left(M^{[p]} w\right)}, \\
\left\|T_{\Phi} f\right\|_{L^{p, q}(w)} \leq C\left\|M_{\widetilde{\Phi}} f\right\|_{L^{p, q}\left((M w / w)^{[p]} M w\right)} .
\end{gathered}
$$

For $k \geq 1$ and $b \in \mathrm{BMO}$, the commutators of potential type integral operator with a BMO function are defined by

$$
T_{\Phi, b}^{k} f(x)=\int_{\mathbb{R}^{n}}(b(x)-b(y))^{k} \Phi(x-y) f(y) d y .
$$

Li [11] gave the two-weight strong type $(p, q)$ inequalities for $T_{\Phi, b}^{k}, 1<p \leq q<\infty$. We can easily get similar results as in Lemmas 6 and 7 from [11]; by Theorem 1, we can obtain some weighted inequalities for the commutators of potential type integral operators; details are omitted.

\section{Proofs of Theorems}

We need some notations and facts. Let $B(t):[0, \infty) \rightarrow$ $[0, \infty)$ be a Young function, that is, a continuous, convex, and increasing function with $B(0)=0$ such that $B(t) \rightarrow \infty$ as $t \rightarrow \infty$. Given a Young function $B$, we define the $B$-average of a function $f$ over a cube $Q$ by

$$
\|f\|_{B, Q}=\inf \left\{\lambda>0: \frac{1}{|Q|} \int_{Q} B\left(\frac{|f(x)|}{\lambda}\right) d x \leq 1\right\} .
$$

In particular, for the Young function $B_{k}(t)=t\left(1+\log ^{+} t\right)^{k}$, $k=1,2, \ldots$, the $B_{k}$-average of a function $f$ over a cube $Q$ is denoted by $\|f\|_{L(\log L)^{k}, Q}$. Define the maximal function associated with the Young function $B_{k}$ as

$$
M_{L(\log L)^{k}} f(x)=\sup _{Q \ni x}\|f\|_{L(\log L)^{k}, Q} \cdot
$$

Pérez [8] obtained that $M_{L(\log L)^{k}} f(y) \approx M^{k+1} f(y)$.

Proof of Theorem 1. Fix $p, p_{0}<p<\infty, p_{0} \leq q \leq \infty$, and let $r=p / p_{0}, s=q / p_{0}$. Then, by duality,

$$
\begin{aligned}
\|T f\|_{L^{p, q}(w)}^{p_{0}} & =\left\|(T f)^{p_{0}}\right\|_{L^{r, s}(w)} \\
& \leq \sup _{g \in L^{r^{\prime}, s^{\prime}}(w),\|g\|_{L^{\prime}, s^{\prime}(w)} \leq 1} \int_{\mathbb{R}^{n}} T f(x)^{p_{0}} g(x) w(x) d x .
\end{aligned}
$$

The last inequality follows since $L^{r^{\prime}, s^{\prime}}(w)$ and $L^{r, s}(w)$ are associate spaces. Fixing one of these $g$ 's, we use (4) to continue with

$$
\begin{aligned}
& \int_{\mathbb{R}^{n}}(T f(x))^{p_{0}} g(x) w(x) d x \\
& \quad \leq C \int_{\mathbb{R}^{n}} S f(x)^{p_{0}} M^{k}(g w)(x) d x
\end{aligned}
$$

$$
\begin{aligned}
& \leq C \int_{\mathbb{R}^{n}} S f(x)^{p_{0}} \frac{M_{L(\log L)^{k-1}}(g w)(x)}{\widetilde{M}(w)(x)} \widetilde{M}(w)(x) d x \\
& \leq C\left\|(S f)^{p_{0}}\right\|_{L^{r, s}(\widetilde{M}(w))}\left\|\frac{M_{L(\log L)^{k-1}}(g w)(x)}{\widetilde{M}(w)(x)}\right\|_{L^{\prime}, s^{\prime}(\widetilde{M}(w))} \\
& =C\|S f\|_{L^{p, q}(\widetilde{M}(w))}^{p_{0}} \| \frac{M_{L(\log L)^{k-1}(g w)(x)}^{\widetilde{M}(w)(x)} \|_{L^{r^{\prime}, s^{\prime}}(\widetilde{M}(w))},}{}
\end{aligned}
$$

where $\widetilde{M}$ is an appropriate maximal operator to be chosen soon. To conclude, we just need to show that

$$
\left\|\frac{M_{L(\log L)^{k-1}}(g w)(x)}{\widetilde{M}(w)(x)}\right\|_{L^{r^{\prime}, s^{\prime}}(\widetilde{M}(w))} \leq C\|g\|_{L^{r^{\prime}, s^{\prime}}(w)},
$$

or equivalently

$$
F: L^{r^{\prime}, s^{\prime}}(w) \longrightarrow L^{r^{\prime}, s^{\prime}}(\widetilde{M}(w))
$$

where

$$
F f=\frac{M_{L(\log L)^{k-1}}(f w)}{\widetilde{M}(w)} .
$$

To do this, we choose $\widetilde{M}$ pointwise bigger than $M_{L(\log L)^{k-1}}$; then, we trivially have

$$
F: L^{\infty}(w) \longrightarrow L^{\infty}(\widetilde{M}(w)) .
$$

Therefore, by Marcinkiewicz's interpolation theorem for Lorentz spaces in [12], it will be enough to show that for some $\varepsilon>0$

$$
F: L^{(r+\varepsilon)^{\prime}}(w) \longrightarrow L^{(r+\varepsilon)^{\prime}}(\widetilde{M}(w)),
$$

which amounts to proving

$$
\begin{aligned}
\int_{\mathbb{R}^{n}} & M_{L(\log L)^{k-1}}(f w)(x)^{(r+\varepsilon)^{\prime}}(\widetilde{M} w(x))^{1-(r+\varepsilon)^{\prime}} d x \\
\leq C & \int_{\mathbb{R}^{n}} f(x)^{(r+\varepsilon)^{\prime}} w(x) d x .
\end{aligned}
$$

But this result follows from [13]: it is shown there that, for $\nu>1$ and $\eta>0$,

$$
\begin{aligned}
& \int_{\mathbb{R}^{n}} M_{L(\log L)^{k-1}}(f)(x)^{\nu^{\prime}}\left(M_{L(\log L)^{k \nu 1+\eta}} w(x)\right)^{1-\nu^{\prime}} d x \\
& \leq C \int_{\mathbb{R}^{n}} f(x)^{\nu^{\prime}} w(x)^{1-\nu^{\prime}} d x .
\end{aligned}
$$

We finally choose the appropriate parameters and weight. Let $\nu=r+\varepsilon, \eta=\varepsilon=([k r]+1-k r) /(k+1)>0$, and pick the weight

$$
\widetilde{M} w=M_{L(\log L)^{[k r]}} w .
$$

This shows that

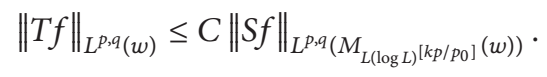

We conclude the proof of (5). 
Proof of Theorem 2. The proof of this theorem proceeds exactly as that of (5) with minor changes. At inequality (23), fixing one of these $g$ 's, we use (6) to continue with

$$
\begin{aligned}
& \int_{\mathbb{R}^{n}}(T f(x))^{p_{0}} g(x) w(x) d x \\
& \quad \leq C \int_{\mathbb{R}^{n}} S f(x)^{p_{0}} M(g w)(x) d x .
\end{aligned}
$$

For $g$ and $w$, we have $M(g w) \leq C M_{c}(g w)$, where $C$ is a constant depending only on the dimension $n$ and $M_{c}$ is the unweighted centered Hardy-Littlewood maximal operator:

$$
M_{c}(f)(x)=\sup _{r>0} \frac{1}{\left|B_{r}(x)\right|} \int_{B_{r}(x)}|f(x)| d x,
$$

in which $B_{r}(x)$ is the ball of radius $r$ centered at $x$. Furthermore,

$$
\begin{aligned}
& M_{c}(g w)(x) \\
& \quad=\sup _{r>0} \frac{1}{\left|B_{r}(x)\right|} \int_{B_{r}(x)} g(y) w(y) d y \\
& \quad=\sup _{r>0} \frac{w\left(B_{r}(x)\right)}{\left|B_{r}(x)\right|} \frac{1}{w\left(B_{r}(x)\right)} \int_{B_{r}(x)} g(y) w(y) d y \\
& \leq M_{w, c} g(x) M w(x),
\end{aligned}
$$

where $M_{w, c}$ is the weighted centered maximal operator. Then, for $\delta>0$,

$$
\begin{aligned}
\int_{\mathbb{R}^{n}}(S f)^{p_{0}} M(g w) d x \\
\leq C \int_{\mathbb{R}^{n}}|S f|^{p_{0}} M_{w, c} g\left(\frac{w}{M w}\right)^{r+\delta-1}\left(\frac{M w}{w}\right)^{r+\delta} w d x \\
\leq C\left\|(S f)^{p_{0}}\right\|_{L^{r, s}\left((M w / w)^{r+\delta} w\right)} \\
\quad \times\left\|M_{w, c} g\left(\frac{w}{M w}\right)^{r+\delta-1}\right\|_{L^{r^{\prime}, s^{\prime}}\left((M w / w)^{r+\delta} w\right)} .
\end{aligned}
$$

To conclude, we just need to show that

$$
\left\|M_{w, c} g\left(\frac{w}{M w}\right)^{r+\delta-1}\right\|_{L^{r^{\prime}, s^{\prime}}\left((M w / w)^{r+\delta} w\right)} \leq C\|g\|_{L^{r^{\prime}, s^{\prime}(w)}},
$$

or equivalently

$$
F: L^{r^{\prime}, s^{\prime}}(w) \longrightarrow L^{r^{\prime}, s^{\prime}}\left(\left(\frac{M w}{w}\right)^{r+\delta} w\right),
$$

where

$$
F f=M_{w, c} f\left(\frac{w}{M w}\right)^{r+\delta-1}
$$

We notice that $M w \leq(M w / w)^{r+\delta} w$ for each $w$. With this, we trivially have

$$
F: L^{\infty}(w) \longrightarrow L^{\infty}\left(\left(\frac{M w}{w}\right)^{r+\delta} w\right)
$$

Therefore, by Marcinkiewicz's interpolation theorem for Lorentz spaces, it will be enough to show that for some $\varepsilon>0$

$$
F: L^{(r+\varepsilon)^{\prime}}(w) \longrightarrow L^{(r+\varepsilon)^{\prime}}\left(\left(\frac{M w}{w}\right)^{r+\delta} w\right)
$$

which amounts to proving

$$
\begin{gathered}
\int_{\mathbb{R}^{n}}\left(M_{w, c} g\left(\frac{w}{M w}\right)^{r+\delta-1}\right)^{(r+\varepsilon)^{\prime}}\left(\frac{M w}{w}\right)^{r+\delta} w d x \\
\leq C \int_{\mathbb{R}^{n}} g^{(r+\varepsilon)^{\prime}} w d x .
\end{gathered}
$$

Taking $\varepsilon=\delta$ and using the well-known fact that $M_{w, c}$ is bounded on $L^{p}(w), 1<p<\infty$, with a constant that depends only on $p$ and $n$, we get (43).

This shows that

$$
\|T f\|_{L^{p, q}(w)}^{p_{0}} \leq C\|S f\|_{L^{p, q}\left((M w / w)^{p / p_{0}+\delta} w\right)}^{p_{0}} .
$$

Taking $\delta=\left[p / p_{0}\right]+1-p / p_{0}>0$, we get (7). This ends the proof of Theorem 2 .

\section{Conflict of Interests}

The authors declare that there is no conflict of interests regarding the publication of this paper.

\section{Acknowledgments}

The authors would like to express their gratitude to the referee for his very valuable comments and suggestions. This study is supported by the Natural Science Foundation of Hebei Province (A2014205069 and A2015403040) and Postdoctoral Science Foundation of Hebei Province (B2013003007).

\section{References}

[1] J. L. Rubio de Francia, "Factorization theory and $A_{p}$ weights," American Journal of Mathematics, vol. 106, no. 3, pp. 533-547, 1984.

[2] D. Cruz-Uribe, J. M. Martell, and C. Pérez, "Extrapolation from $A_{\infty}$ weights and applications," Journal of Functional Analysis, vol. 213, no. 2, pp. 412-439, 2004.

[3] G. P. Curbera, J. García-Cuerva, J. M. Martell, and C. Péreza, "Extrapolation with weights, rearrangement-invariant function spaces, modular inequalities and applications to singular integrals," Advances in Mathematics, vol. 203, no. 1, pp. 256-318, 2006.

[4] D. Cruz-Uribe and C. Pérez, "Two weight extrapolation via the maximal operator," Journal of Functional Analysis, vol. 174, no. 1, pp. 1-17, 2000.

[5] C. Fefferman and E. M. Stein, "Some maximal inequalities," The American Journal of Mathematics, vol. 93, pp. 107-115, 1971.

[6] J. M. Wilson, "Weighted norm inequalities for the continuous square function," Transactions of the American Mathematical Society, vol. 314, no. 2, pp. 661-692, 1989.

[7] C. Pérez, "Weighted norm inequalities for singular integral operators," Journal of the London Mathematical Society: Second Series, vol. 49, no. 2, pp. 296-308, 1994. 
[8] C. Pérez, "Sharp estimates for commutators of singular integrals via iterations of the Hardy-Littlewood maximal function," Journal of Fourier Analysis and Applications, vol. 3, no. 6, pp. 743-756, 1997.

[9] A. K. Lerner, "Weighted norm inequalities for the local sharp maximal function," Journal of Fourier Analysis and Applications, vol. 10, no. 5, pp. 465-474, 2004.

[10] C. Pérez, "Two weighted inequalities for potential and fractional type maximal operators," Indiana University Mathematics Journal, vol. 43, no. 2, pp. 663-683, 1994.

[11] W. Li, "Two-weight norm inequalities for commutators of potential type integral operators," Journal of Mathematical Analysis and Applications, vol. 322, no. 2, pp. 1215-1223, 2006.

[12] C. Bennett and R. Sharpley, Interpolation of Operators, Academic Press, New York, NY, USA, 1988.

[13] C. Pérez, "On sufficient conditions for the boundedness of the Hardy-Littlewood maximal operator between weighted $L^{p_{-}}$ spaces with different weights," Proceedings of the London Mathematical Society: Third Series, vol. 71, no. 1, pp. 135-157, 1995. 


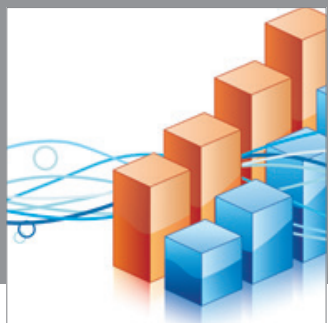

Advances in

Operations Research

mansans

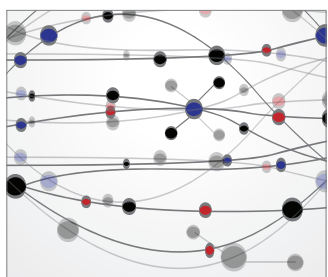

The Scientific World Journal
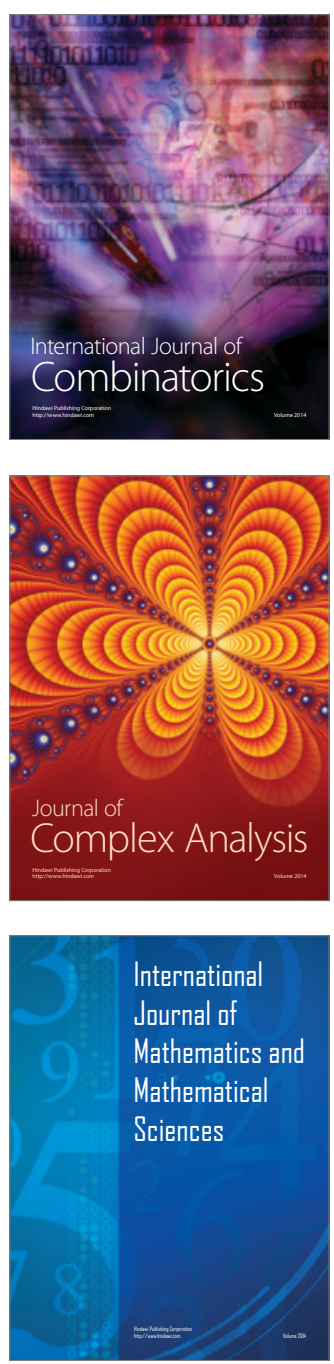
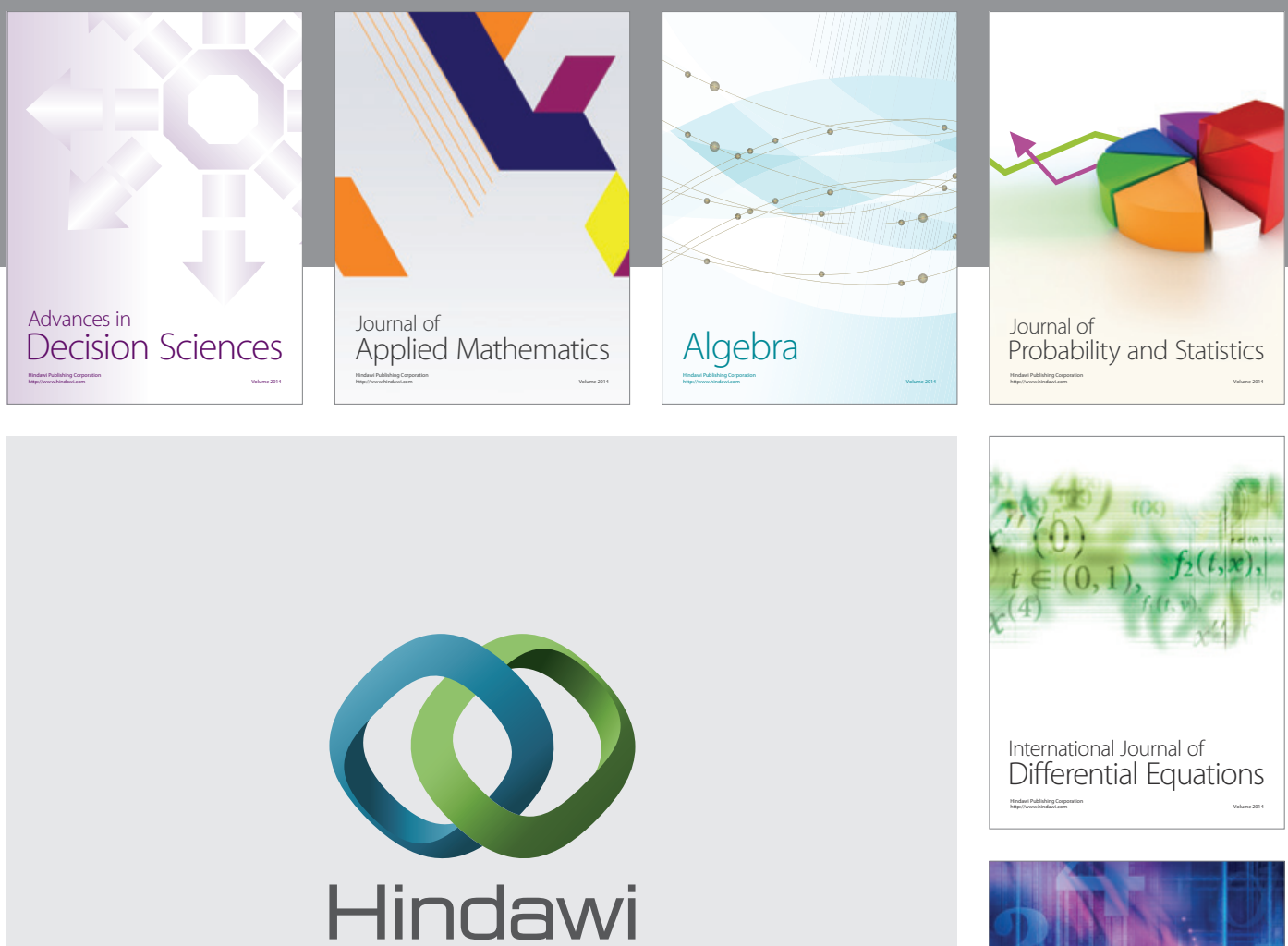

Submit your manuscripts at http://www.hindawi.com
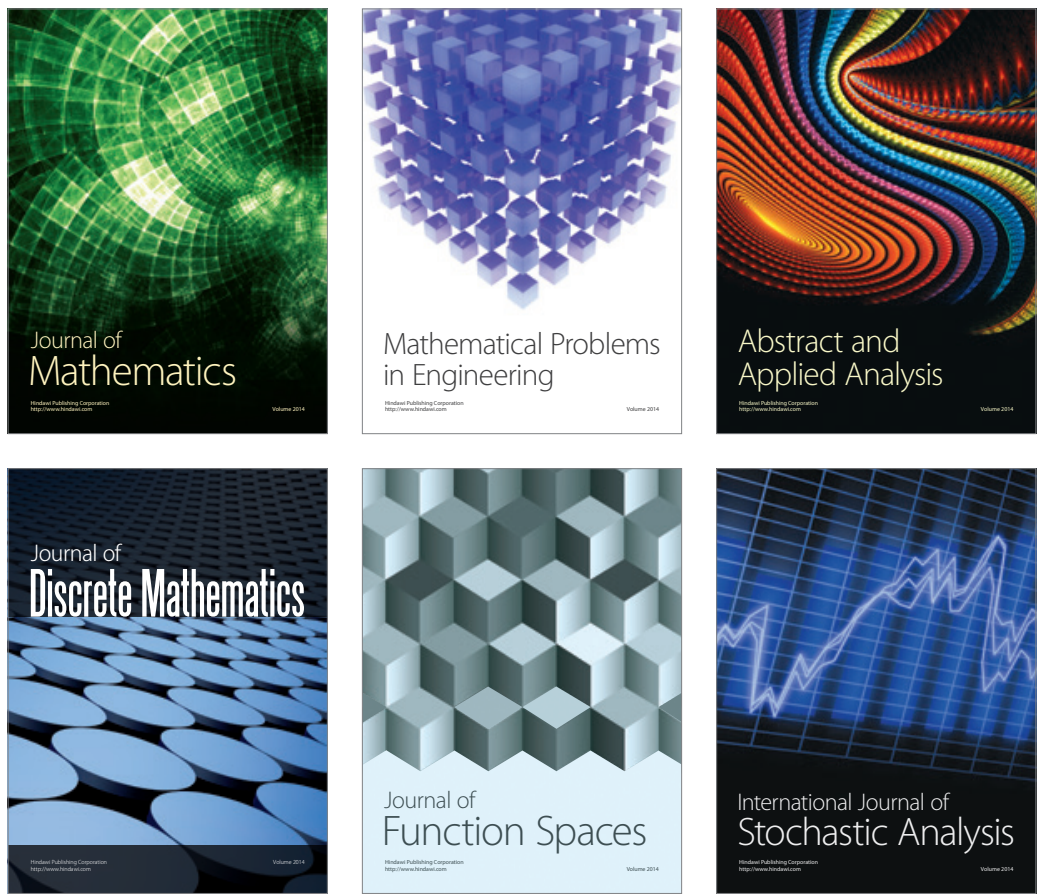

Journal of

Function Spaces

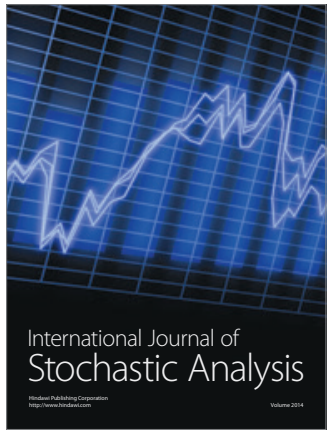

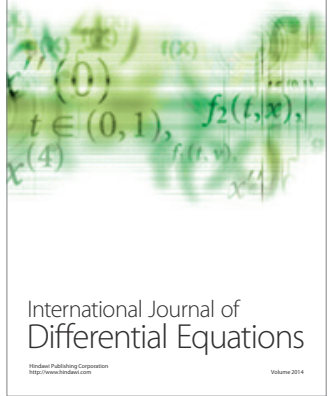
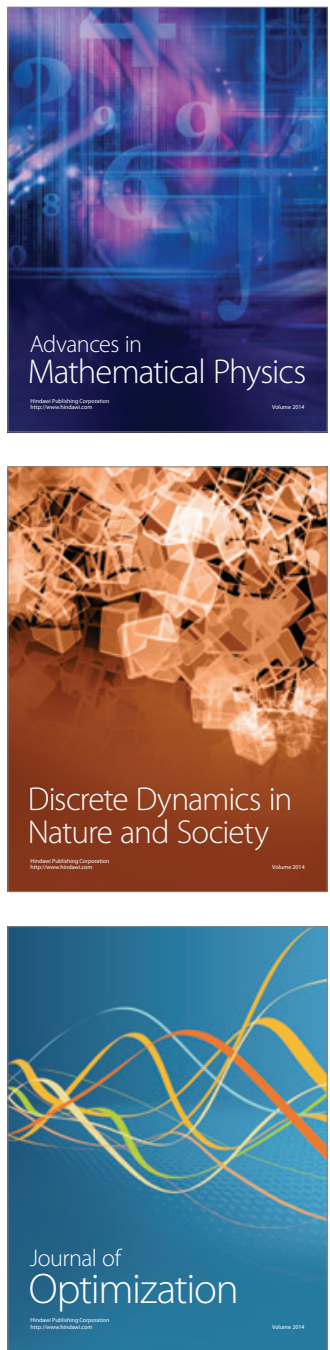